\title{
Branching Morphogenesis: From Cells to Organs and Back
}

\section{Amanda Ochoa-Espinosa and Markus Affolter}

Biozentrum der Universität Basel, Basel, Switzerland

Correspondence: markus.affolter@unibas.ch

\section{SUMMARY}

Many animal organs, such as the lung, the kidney, the mammary gland, and the vasculature, consist of branched tubular structures that arise through a process known as "branching morphogenesis" that results from the remodeling of epithelial or endothelial sheaths into multicellular tubular networks. In recent years, the combination of molecular biology, forward and reverse genetic approaches, and their complementation by live imaging has started to unravel rules and mechanisms controlling branching processes in animals. Common patterns of branch formation spanning diverse model systems are beginning to emerge that might reflect unifying principles of tubular organ formation.

\section{Outline}

1 Introduction

2 Branching in different organ systems

3 The tracheal system of Drosophila

4 The vasculature

5 The lung

6 The kidney

7 The mammary gland
8 Regionalization of branches during outgrowth: Generation of tip versus stalk

9 Control of cell behavior during the branching process: A comparison

10 Branch outgrowth: Reiteration of a basic process?

11 Methods of choice to understand branching morphogenesis

References

Editors: Patrick P.L. Tam, W. James Nelson, and Janet Rossant

Additional Perspectives on Mammalian Development available at www.cshperspectives.org

Copyright (C 2012 Cold Spring Harbor Laboratory Press; all rights reserved; doi: 10.1101/cshperspect.a008243

Cite this article as Cold Spring Harb Perspect Biol 2012;4:a008243 


\section{INTRODUCTION}

Branched structures in biological and nonbiological systems have fascinated human beings and stimulated scientists over decades. In the past 20 years, the combination of molecular biology, forward and reverse genetic approaches, and their complementation by live imaging has started to unravel rules and mechanisms controlling branching processes in animals. Branching underlies the formation of numerous organs, including the nervous system, the respiratory system, and many internal glands, as well as the vasculature (see Fig. 1 for a collection of examples of branched organs). Each of these organs has its own evolutionary history, and to decipher its developmental program represents a major challenge for biologists. Once a better understanding of the branching process of various organs is achieved, diverse questions regarding their underlying similarities can be addressed.

Because many of the organ systems we discuss here are also covered in other articles in this collection, we deal exclusively with the process of branching morphogenesis and touch on cell differentiation only when necessary. Rather than putting together a comprehensive review of branching morphogenesis, we highlight a few aspects of particular interest and illustrate the latter with well-defined examples. We emphasize specific experimental strategies used to decipher certain cellular aspects of shape development, with the hope that similar in-depth experiments in other systems will be equally rewarding. We limit our discussions to the Drosophila tracheal system as an invertebrate model for branching and discuss angiogenesis as well as lung, kidney, and mammary gland branching in vertebrates (Fig. 1A-E). It has been pointed out that "branching morphogenesis" also occurs in the nervous system; individual cells branch extensively and most often in a highly reproducible manner (see Fig. 1F). Much can be learned from neural branching, although the process occurs at the singlecell level; however, because of lack of space, we have limited these discussions and refer the reader to other reviews written on this topic ( $\mathrm{Lu}$ and Werb 2008; Grueber and Sagasti 2010; Jan and Jan 2010; Gibson and Ma 2011). Organ branching morphogenesis has been covered in many excellent reviews (for examples, see below); here, we concentrate on cellular activities underlying branching to highlight similarities and differences between the cellular strategies used in different systems.

\section{BRANCHING IN DIFFERENT ORGAN SYSTEMS}

When reflecting about the branched organs depicted in Figure 1, major differences can be put forward; whereas some branched systems ramify throughout the entire body and interact with several other organs and tissues (e.g., the vasculature in vertebrates and the tracheal system in flies), others are confined more locally (lung, kidney, mammary gland) and branch in a confined space. The development of
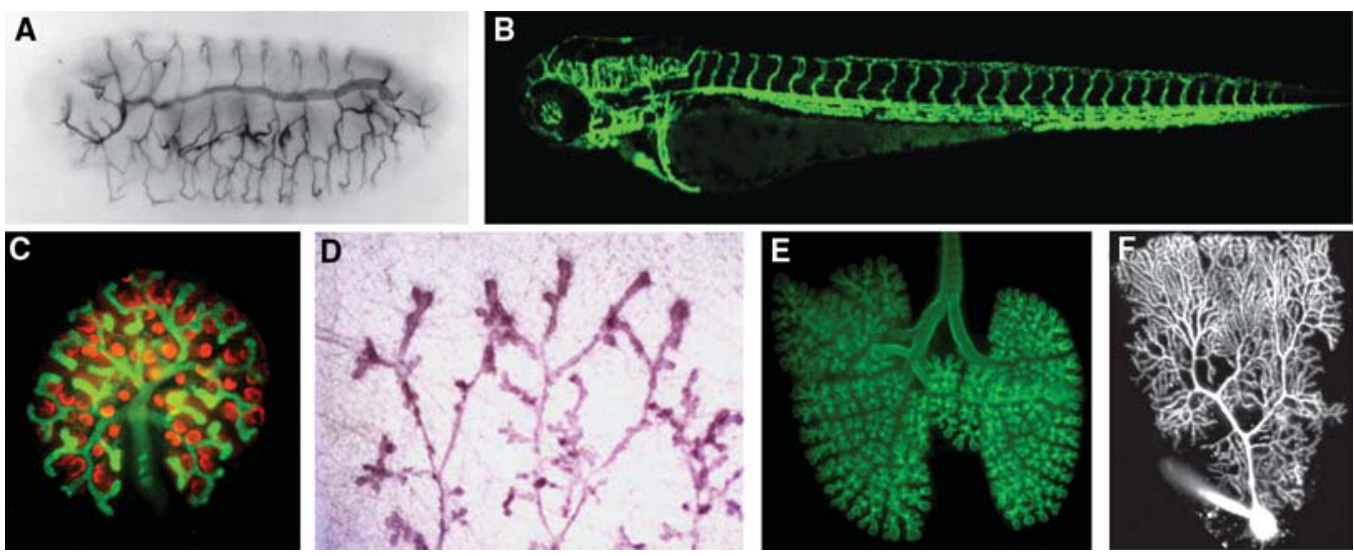

Figure 1. Branched animal organs. (A) Drosophila embryonic tracheal system stained with the tracheal luminal antigen mAb2A12, reproduced with permission from Development (Samakovlis et al. 1996a) (http://dev.biologists. org/content/122/5/1395). (B) Three-day-old zebrafish embryo vascular system expressing EGFP under the control of the endothelial specific promoter of the $f l k 1$ gene (TG:flk1:EGFP). (C) Developing mouse kidney stained for Wilm's tumor 1 antigen (red, developing glomeruli) and calbindin (green, ureteric tree). (Photo courtesy of Kenneth Walker and John Bertram.) (D) Mammary gland of a virgin rat (Schedin et al. 2007). (From Schedin et al. 2007; adapted, with kind permission, from Springer Science+Business Media, J Mammary Gland Biol Neoplasia (C) 2007.) (E) Whole mount of an embryonic day 14.5 (E14.5) mouse lung stained for E-cadherin to show the airway epithelium. (Photo courtesy of Ross J. Metzger.) (F) Two-photon fluorescence image of Purkinje cell filled with a fluorescent dye. (Photo courtesy of Yo Otsu and Stéphane Dieudonné.) 
the vasculature and the tracheal system has to obey the body plan of the organism in which it develops in order to access each and every organ. Other branched structures must "only" fulfill their own needs with their branching pattern; the lung needs to branch in concert with the vasculature, but does not have to take into account other structures such as bones or muscles. These differences have consequences at the level of controlling where and how to branch, and we briefly outline in the following section what is known regarding the formation of the three-dimensional (3D) architecture of the branched organs discussed here.

\section{THE TRACHEAL SYSTEM OF Drosophila}

The larval tracheal system of Drosophila melanogaster is one of the best studied branched structures (Ghabrial et al. 2003; Uv et al. 2003; Affolter and Caussinus 2008; Andrew and Ewald 2010; Schottenfeld et al. 2010; Maruyama and Andrew 2012). The tracheal network is established during embryonic development from groups of epithelial cells that have been determined to become part of the tracheal system. After the formation of 10 sac-like invaginations of approximately 80 epithelial cells on either side of the embryo, branches grow out from these invaginations in the complete absence of cell division. Branch formation relies on cell migration, cell rearrangements, and cell shape changes, which occur in a highly organized and reproducible manner. Branchless (Bnl), a fibroblast growth factor (Fgf) ligand, acts at the top of a hierarchy of cellular events and controls and coordinates branch formation. Bnl is expressed in epidermal or mesodermal cells of target areas/tissues in the vicinity of the tracheal sac (Sutherland et al. 1996). Tracheal cells sense the sources of Bnl through the Fgf receptor Breathless (Btl) on their basal side (Klambt et al. 1992; Sutherland et al. 1996), which results in the formation of numerous filopodia and ultimately in the migration of a few cells away from the sac-like invagination. This migration generates interconnected, bud-like extensions because the epithelial cells are attached to each other via adherens junctions and migrating cells thus pull along their neighbors. The cells in many of these buds are further rearranged to generate fully extended, finer branches by means of two additional cell behaviors trigged by Bnl: the selection of tip/stalk cells and cell intercalation. Cells with the highest Btl receptor activity assume tip positions, whereas cells with lower activity follow tip cells and ultimately form stalk cells (Fig. 2A) (Ghabrial and Krasnow 2006). This cell competition appears to rely on Notch/Delta signaling; cells with high levels of Btl activity produce more Delta (Dl), which leads to Notch $(\mathrm{N})$ activation in neighboring cells. This tip/stalk cell distinction confers the extending branch with a polarized aspect along the axis of outgrowth; distal tip cell migration generates a tensile stress in proximal stalk cells and eventually triggers their intercalation, elongating the branch along its axis of outgrowth (Caussinus et al. 2008). Stalk cells elongate even further upon intercalation. Thus, force generation in the tip cells, in addition to cell intercalation, generates elongated cell shapes, again as a consequence of Bnl-driven tip cell migration. Therefore, Bnl-Btl signaling controls tip cell selection
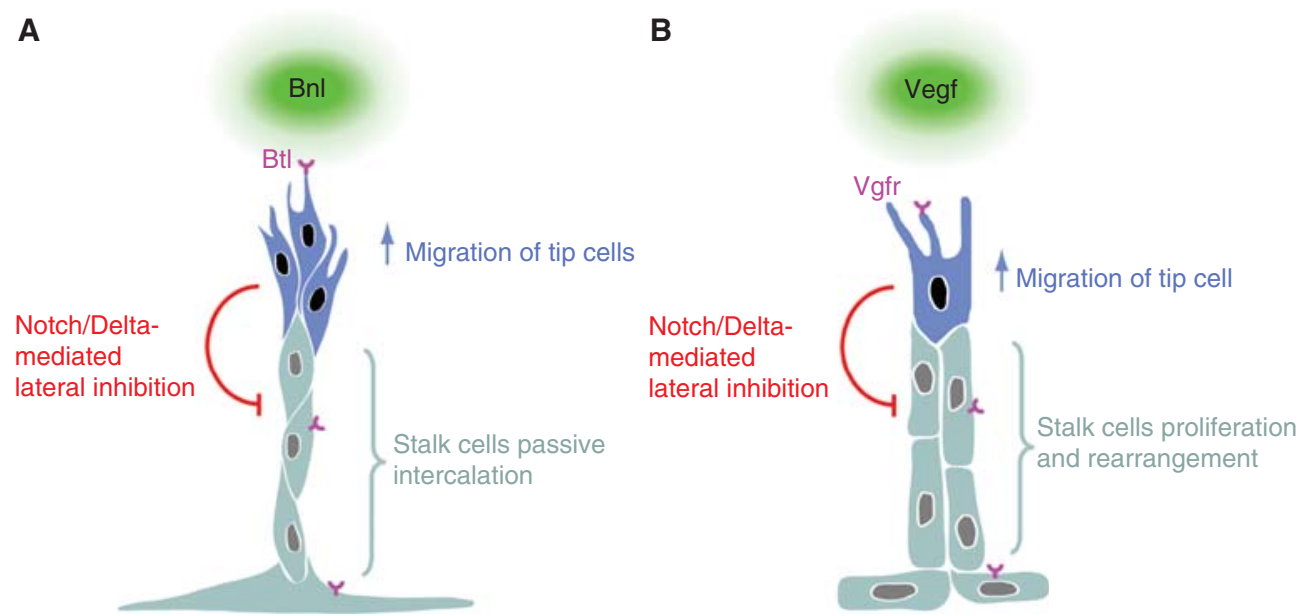

Figure 2. Branch regionalization and outgrowth. $(A)$ Tracheal branching, and $(B)$ vertebrate angiogenesis. Both Bnl and Vegf ligands elicit a migratory behavior through their respective receptors Btl and Vgfr. In both instances, the cells with the highest receptor activity become tip cells and produce more Delta, leading to the activation of Notch in neighboring stalk cells, which, in turn, inhibit the tip cell fate. Although Fgf signaling in the trachea triggers migration in the tip cells and, as a consequence, passive stalk cell intercalation, Vegf signaling in the vasculature not only triggers tip cell migration but also actively regulates the proliferation and rearrangement of stalk cells. 
and cell migration, and, as a consequence, stalk cells intercalate and extensively elongate to generate branches that extend to and reach the target tissue. Interestingly, stalk cells do not need to be responsive to Bnl signaling to undergo intercalation and/or elongation, because a single Btlpositive cell can lead and form a normal branch (Ghabrial and Krasnow 2006; see also Cabernard and Affolter 2005).

The $3 \mathrm{D}$ architecture of the branching pattern in the embryonic tracheal system is thus governed via the control of the expression pattern of the $b n l$ gene; local sources of Bnl trigger locally restricted stereotyped cell behaviors, ultimately leading to the formation of a branch reaching the signal source. Nonetheless, even branch-specific architectural aspects such as tip stalk cell subdivision, cell intercalation, and cell shape are triggered by Bnl. It is worth mentioning, however, that the highly organized pattern of the trachea seen in developing embryos is to a large extent also due to topological constraints, that is, to the alignment and the restriction of space for tracheal cell migration by other organs that are also spatially organized in a highly ordered manner (Fig. 3B) (Franch-Marro and Casanova 2000; Casanova 2007).

To generate an interconnected tracheal network capable of performing its function in gas transport, many further processes have to occur, such as regulation of tube diameter, growth of the tubes, lumen formation in terminal cells, fusion of adjacent metamers, gas filling, and tube maintenance (Samakovlis et al. 1996b; Luschnig et al. 2006; Wang et al. 2006; Tsarouhas et al. 2007; Gervais and Casanova 2010; Ghabrial et al. 2011). In a recent screen for tube morphogenesis and branching genes in the tracheal system, Krasnow and colleagues estimated that more than 200 patterning and morphogenesis genes are required to build the relatively simple tracheal system in Drosophila (beyond the "housekeeping" genes required in most cells of the organism) (Ghabrial et al. 2011). It will be interesting to find out how many of them are used during the branching process and redeployed in later steps, and how many are used exclusively for branching and functional aspects, respectively.

\section{THE VASCULATURE}

The most ramified branched organ (apart from the nervous system) in any vertebrate animal is the vasculature. The vasculature ensures the proper transport and distribution of relevant molecules and cells to every organ. The initial vessels are generated via a process called "vasculogenesis," that is, the de novo formation of vessels from mesodermderived endothelial precursors. The vessels laid down by vasculogenesis often form an initial circuit, allowing blood to flow away from the heart and back to it (Swift and Weinstein 2009). However, because this network is not
A

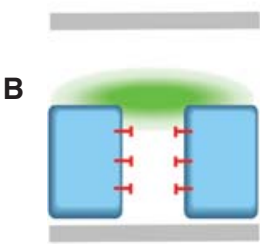

C

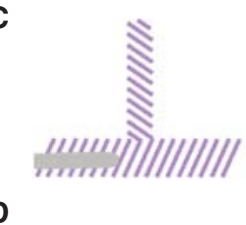

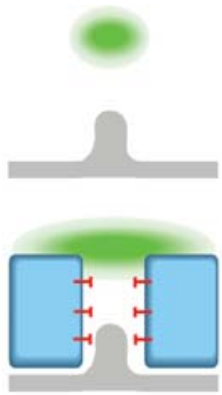

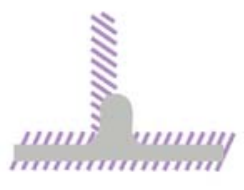

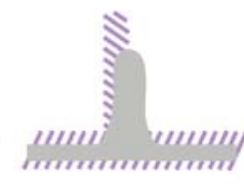

-Substrate guided

-Intussusception

-Localized attractive source

-Attractive source

-Physical/repulsive constraints
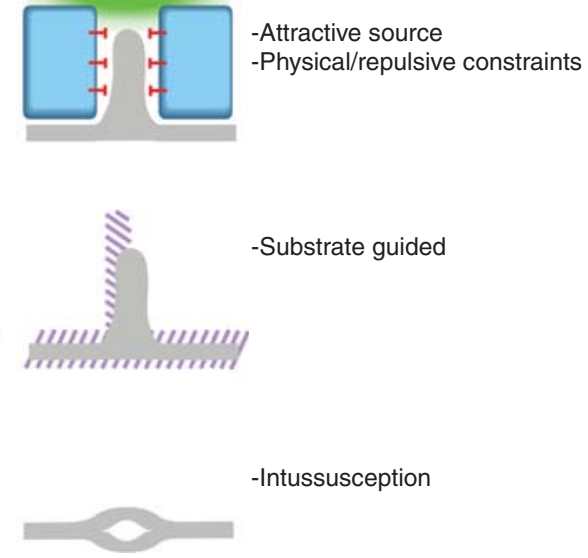

Figure 3. Branching strategies. Organ branching can be dissected into a few basic strategies. None of the branching events described in this review follow one single scheme but, rather, a combination of them. However, the identification within each organ of these prime processes can help us to understand and experimentally examine their development. (A) Migration toward a signaling source (green). (B) Migration toward a signaling source (green) constrained by repulsive signals (red block-arrows) or physical obstacles (blue). (C) Migration following an underlying patterned structure (purple stripes). (D) Intussusception: splitting of a branch into finer branches. 
generated by branching morphogenesis and assembles to a large extent from individual angioblasts in situ, we do not further discuss how its architecture is achieved. Nevertheless, these initial vessels serve as the "substrate" for the formation of most of the fine, branched vascular networks that appear during later developmental stages. In many cases, these vessels arise through branching morphogenesis (i.e., the formation of new branches out of existing ones), but the process of their formation is generally referred to as "sprouting angiogenesis" (Risau 1997; Geudens and Gerhardt 2011; Wacker and Gerhardt 2011) (a second mechanism, "intussusceptive angiogenesis," is discussed below). Growth of new vessels requires endothelial cell (EC) division, directional migration, cell rearrangements, and cell shape changes, and, similar to the trachea, it is mostly stereotyped in the developing early embryo. Sprouting involves the formation of tip cells that form numerous filopodial extensions and explore the environment to react to several positive and negative guidance cues; eventually, tip cells migrate under the control of, or toward, regions of pro-angiogenic factors. One of the key molecules inducing sprouting is vascular endothelial growth factor A (Vegf-A). In the early mouse postnatal retina, tip cell migration depends on a gradient of Vegf-A, whereas stalk cell proliferation requires a certain threshold level of Vegf-A. Vegf-A is secreted by an underlying network of astrocytes, which thus prefigures the branching pattern (Gerhardt et al. 2003). Vegf-A signals via vascular endothelial growth factor receptor 2 (Vegfr2), but other Vegf ligands and their respective receptors also play important roles in angiogenesis (Leung et al. 1989; Ferrara et al. 2003; Ruhrberg 2003; Coultas et al. 2005; Lohela et al. 2009).

Because tip cells play such a pivotal role in vascular branching morphogenesis, key questions are how tip cells are selected from the endothelial layer of a preexisting vessel to initiate sprouting and thus trigger branch formation at defined positions, and how tip cells lead and guide the newly formed vessel branch. Tip cell selection is linked to the ligand Delta-like 4 (Dll4) and its receptor Notch 1 (Fig. 2B) (Shutter et al. 2000; Liu et al. 2003; Williams et al. 2006; Hellstrom et al. 2007; Leslie et al. 2007; Lobov et al. 2007; Siekmann and Lawson 2007; Suchting et al. 2007). The Dll4 gene is a target of Vegf signaling in endothelial cells and is preferentially induced in cells nearest to a source of Vegf (because of the highest level of ligand bound to receptor). Cells with the highest level of Dll4 develop a tip cell phenotype and inhibit their immediate neighbors via Dll4Notch 1 activation from adopting the same phenotype, thus pushing them toward the stalk cell phenotype. Although the details of the dynamic interaction between tip and stalk cells is poorly understood, this regulatory module is at the core of vascular branching morphogenesis. Recent studies showed that tip cell selection (and thus branch formation) involves many other molecules, which in most cases limit the angiogenic potential to specific regions either via the regulation of Vegf signaling or via the Notch/Delta pathway (Kim et al. 2011; Zygmunt et al. 2011). Particularly interesting is the recent finding that axonal guidance molecules influence branching of the vasculature via their respective receptors, which are expressed in endothelial cells (Lu et al. 2004; for review, see Adams and Eichmann 2010). Further studies regarding the role and molecular function of the axon guidance molecules on the formation and function of the vasculature promise to add much to our current understanding of vascular branching.

Although Vegfs play a crucial and instructive role in branching of the developing vasculature, similar to Bnl in tracheal branching in Drosophila, the final branching pattern of the vasculature can be influenced or controlled by several other processes and molecular pathways. In many instances, the blood vessels follow structures built by another branching program, such as the lung branching program used by the lung vasculature or the pattern of the peripheral nerves used by the vasculature in the embryonic skin (Fig. 3C). Thus, it seems that in many cases, branch points in the vasculature are determined by a preexisting structure, and the underlying branching logic might be attributed to another organ system. This does not mean that in these cases Vegfs might not play a role as sprouting factors (e.g., embryonic nerves are the source of Vegf) (Mukouyama et al. 2005), but that the building plan has been "borrowed" from an underlying tissue. A beautiful example of this mechanism is the tightly choreographed development of the string of endothelial cells forming the parachordal chain (PAC) during zebrafish lymphatic system development. In this three-tiered system, a group of muscle pioneers localized along the horizontal myoseptum release Netrin la and guide the migration of receptor-expressing RoP motoneuron axons along it. In turn, the aligned RoP axons establish the migration path of the PAC (Lim et al. 2011).

Many of the vascular networks observed in internal organs of adult organisms might be established via a primordial capillary plexus, which then grows and remodels through "intussusceptive angiogenesis," the splitting of preexisting vessels into finer ones, thereby enlarging and refining a preformed branching network (Fig. 3D) (Burri et al. 2004; Makanya et al. 2009). The branch points in such a situation cannot be explained with the classical view of branching morphogenesis, because they do not arise via sprouting angiogenesis (via the de novo formation of a branch at a given position), but rather via splitting mechanisms.

Because most branching patterns observed in the mature vasculature have not been sufficiently analyzed during 
development, it is too early to formulate a "branching morphogenesis program" for the entire vascular network. Deciphering the final branching logic of the vasculature will also require an understanding of the patterning of the underlying substrates, highlighting the coordinated establishment of the vasculature with regard to all other organs in the body.

\section{THE LUNG}

The lung consists of two intertwined and highly branched tree-like tubular systems-one conducting air and the other, the vasculature, conducting blood cells and serum (for review, see Morrisey and Hogan 2010). Recently, the $3 \mathrm{D}$ branching pattern of the mouse lung has been described by reconstructing its developmental history in vivo, revealing that the airway branching pattern is remarkably stereotyped (Metzger et al. 2008). After the formation of the initial lung buds, the airways are generated by three geometrically distinct modes of branching, each of which gives rise to a different arrangement of branches: domain branching, planar bifurcation, and orthogonal bifurcation. These three modes are used in three different fixed sequences in a stereotyped pattern to generate the characteristic mouse bronchial tree. Each sequence begins with domain branching, but once this mode switches to orthogonal bifurcation, the switch is permanent. Branches that form by domain branching can also undergo planar bifurcation, followed by a subsequent round of domain branching off their sides as well as planar bifurcation at the tip. The lineage of each branch can be traced, and the pattern of where and when each branching mode is used is stereotyped, suggesting that the branching program is hardwired and genetically controlled in time and space. The programmed use of these different branching modes and the sequences in which they are used presumably ensures that the 3D space is filled in the most efficient manner, allowing for dense packing of tubes and maximizing surface area in a given volume.

Although relatively little is known regarding the molecular control of this lung branching program, Krasnow and Metzger propose that the three branching subroutines are controlled genetically by proximal-distal and circumferential patterning. These control mechanisms may include a "periodicity generator," which determines where and when side branches form during domain branching, and so-called "bifurcator" and "rotator" mechanisms, which determine when branches bifurcate and whether bifurcation is planar or orthogonal, respectively. All of these predicted functions would impinge on a more general "branch generator," a cellular routine that initiates branch formation and outgrowth (Metzger et al. 2008).
Many signaling pathways have been implicated in lung formation, and among them a key role has been attributed to Fgf signaling (Fig. 4). The single-cell-layered lung epithelium is surrounded by mesenchymal cells throughout the branching process, and reciprocal interactions between the two cell populations control and regulate branching. Fibroblast growth factor 10 (Fgf10) is dynamically expressed in the distal mesenchyme around epithelial bud tips and is essential for bud formation and outgrowth (Bellusci et al. 1997b; Sekine et al. 1999; De Moerlooze et al. 2000). Fgf10 binds to fibroblast growth factor receptor $2 b$ (Fgfr2b), which is expressed in the epithelial cells and is also required for budding. Several genes are expressed at higher levels in the epithelial bud tips than in the stalk, including sprouty 2

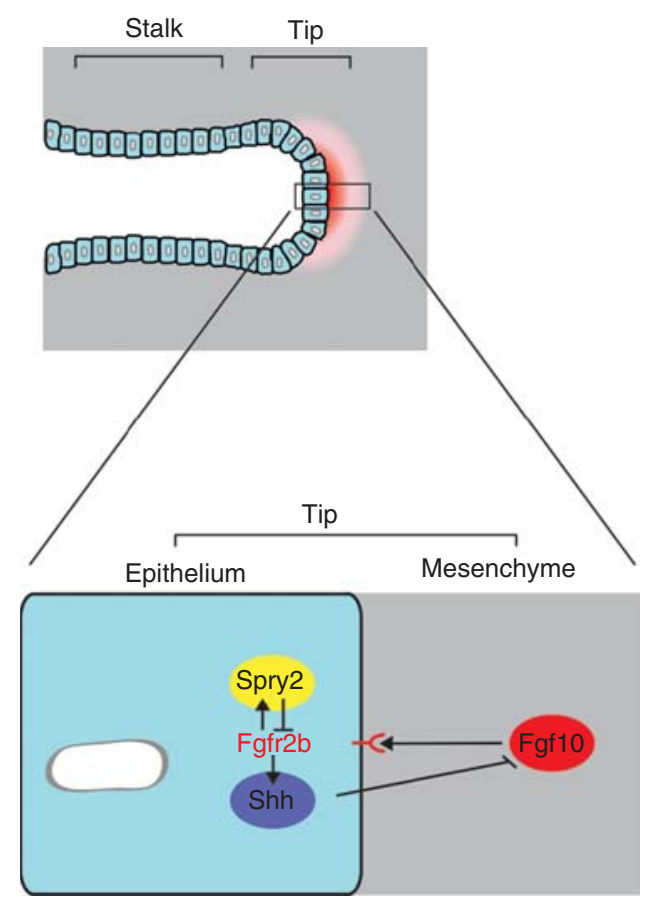

Figure 4. Molecular regulation of lung branching morphogenesis. A distal organizer and signaling center is located at the tip of the primary lung buds. At the core of this center is a set of reciprocal interactions between the bud epithelium and its surrounding mesenchyme. Fgf signaling promotes lung bud outgrowth; Fgf10 is expressed in the mesenchyme and signals to the epithelium through its receptor Fgfr2b. Fgf signaling increases Spry2 expression, which then antagonizes Fgf signaling in the epithelium. Additionally, Fgf signaling-induced Shh signals from the epithelium to the mesenchyme to negatively regulate $F g f 10$ expression in that tissue. Bmp4, on the other hand, seems to have a branching-promoting activity in the mesenchyme and an Fgf signaling down-regulation function within the epithelium, suggesting that correct Bmp4 levels are essential for lung development (not shown). The reciprocal interactions between a branching epithelium and the surrounding mesenchyme seem to be the organizing structural basis of many other budding organs such as the limb bud and the kidney. 
(Spry2), bone morphogenetic protein 2 (Bmp2), and Bmp4, as well as sonic hedgehog (Shh) (Bellusci et al. 1996; Lebeche et al. 1999; Tefft et al. 1999; Mailleux et al. 2001; Eblaghie et al. 2006). Spry2 negatively regulates Fgf signaling in the epithelial cell layer, whereas Shh, a secreted signaling molecule induced by Fgf signaling, regulates the progression of epithelial branching by negatively regulating Fgf10 expression through its receptor Patched (Ptc) in the mesenchyme (Bellusci et al. 1997a). It is very likely that the cross-regulation of Fgf and Shh is at the core of the branching process and thus provides a self-regulatory module of considerable interest (similar to the situation in limb bud outgrowth) (see Affolter et al. 2009; Zeller et al. 2009). Many other signaling pathways have been implicated in regulating lung development during branching, including Wnt and Netrin (Bellusci et al. 1996; Mucenski et al. 2003; Liu et al. 2004; Shu et al. 2005). Further genetic analyses should uncover more precisely the role of these pathways in the branching process, how branching patterns are controlled, and which gene functions underlie the predicted genetic regulators (periodicity, bifurcator, and rotators).

Although many genes have been linked to lung branching, the underlying cell behaviors that must be controlled by these gene products and eventually lead to the architectural changes in the lung epithelium during branch formation are not fully known. For instance, it is not known whether bud outgrowth and elongation are the result of directional cell migration such as in the tracheal system and during angiogenic sprouting, due to the lack of an in vivo system in which cell behavior can be assessed and quantified in real time.

More recent studies, however, have provided insights regarding the control of cell behavior during lung development. During the early development of the lung, tubes change shape by increasing their length more than their circumference. This is due to a bias in the orientation of cell division, with a large proportion of lung epithelial cells dividing parallel to the airway longitudinal axis. The bias in the orientation of the division plane is set by regulating extracellular signal-regulated kinase 1 (Erk1) and Erk2 signaling, which, in turn, influences the control of mitotic spindle orientation; cells that divide parallel to the airway longitudinal axis have lower levels of Erk1/2 signaling. Sprouty genes, which encode negative regulators of Fgf10 signaling, are involved in the Erk1/2 down-regulation, and in their absence, cell division planes are oriented randomly in the stalk, leading to airways that are wider and shorter than normal (Tang et al. 2011). The findings of this study begin to unravel how the regulation of a signaling pathway (Erk1/2) leads to a specific cell behavior (oriented cell division) that at the end impinges on tissue-level morphogenesis (tube elongation).
Another recent study points toward an important role of blood vessels in the control of the branching routines described during mouse lung development. Using different strategies to eliminate the lung vasculature, Lazarus et al. (2011) reported that although epithelial branching per se occurred at a normal rate, branching stereotypy was dramatically disturbed following vascular ablation. In particular, it appeared that the rotator function was impaired such that branches formed parallel to or at a shallow angle instead of perpendicular to the axis (Lazarus et al. 2011). One promising molecular explanation for the phenotypes observed is the fact that Fgf10 expression was perturbed in vessel ablated lungs, and instead of being distributed at focal points juxtaposed to branch tips, it appeared poorly focused throughout the mesenchyme. This result suggests that nearby vessels act to restrict FgF10 expression spatially in the lung mesenchyme and therefore have an essential role in lung $3 \mathrm{D}$ branching patterning.

\section{THE KIDNEY}

Similar to the lung, the kidney collecting ducts form via branching of an epithelial cell layer surrounded by mesenchymal cells (for review, see Michos 2009; Costantini and Kopan 2010). The process is started with the formation of the ureteric bud (UB), which originates from the caudal end of the Wolffian duct and invades the metanephric mesenchyme. Starting from the UB, the UB branching process appears as a reiteration of events including increased proliferation at the distal tip, bifurcation, and subsequent stalk elongation. At the onset of these events, increased cell proliferation leads to a characteristic swelling of the tip-the ampulla. Proliferating ampulla cells extend the tip bilaterally, which results in symmetrical bifurcation or branching; the newly formed stalks elongate until further branching is initiated at the tip. In the mouse, 10 subsequent rounds of ureteric branching occur in a coordinated but not synchronous manner. Tip branching might occasionally result from trifurcation events that later remodel to appear as bifurcations (Watanabe and Costantini 2004). Cell tracing experiments in cultured kidney explants showed that as tip cells divide, some of the daughter cells remain at the tip, while others are left behind and contribute to the forming stalk (Shakya et al. 2005). In a process unrelated to branching morphogenesis, the collecting duct tips ultimately induce the formation and connect to nephrons, making up the particular functional structure of the kidney (for review, see Costantini and Kopan 2010).

Genetic analyses have uncovered several important regulators of kidney branching. The most crucial role in UB induction and branching has been attributed to the secreted signaling molecule glial cell-line-derived neurotrophic 
factor (GDNF) and its receptor rearranged during transfection (Ret). Genetic ablation of either Gdnf or Ret causes renal aplasia in most mutant mice embryos (Costantini and Shakya 2006). Although Ret is expressed in the epithelium, the GDNF ligand is specifically produced in, and secreted from, mesenchymal cells adjacent to the bud (Costantini 2006). The prominent role of GDNF in UB branching is supported by the genetic requirement of a number of genes that either positively or negatively regulate GDNF expression or Ret signaling, and by a large number of genetic interaction studies (for review, see Costantini and Kopan 2010). Strikingly, Fgf signaling can substitute for GDNF/ Ret signaling in vivo under certain conditions (e.g., in Spry/GDNF or Spry/Ret double mutants) (see Michos et al. 2010), showing that some aspects of UB branching can be triggered by other Receptor Tyrosine Kinases (RTKs), including Fgfrs. Thus, it appears that a conserved gene network involving RTKs and their downstream ETS transcription factors promotes and controls renal branching morphogenesis. However, it remains a puzzle how the spatial, 3D aspects of the renal branching pattern are regulated in vivo by GDNF and these other factors. Kidney and lung branch formation appear to be very similar, and in both cases, it remains to be elucidated how the spatial control of GDNF and Fgf signaling is achieved in the kidney and the lung, respectively.

Very little is known regarding the cellular events involved in UB branching and how distinct cell activities (such as directed cell migration and cell competition, which are key activities in the trachea and the vasculature) contribute to the branching process. Although directed cell movement occurs in the formation of the UB from the Wolffian duct (Chi et al. 2009), it is not known whether this particular process also contributes to the later branching events. In contrast to the early stages of UB formation, the UB tips are composed of a single-layered epithelium during subsequent branching events (Chi et al. 2009). Because mitotic cells are diffusely distributed in the ampulla, branching is unlikely to be driven by localized proliferation (Michael and Davies 2004). Based on the chemo-attractive properties of GDNF (Tang et al. 2002), it has been suggested that UB branching is induced and controlled by the attraction of UB tips toward local sources of GDNF (Sariola and Saarma 2003). Methods to manipulate the pattern of GDNF expression locally and precisely will be needed to better test this model. Furthermore, high-resolution live imaging and quantitative image analysis will be important to support or refute this model. However, and similar to the lung, elongation and narrowing of ducts are better understood at the cellular level than branching per se. The elongation of the collecting duct is also driven by oriented cell division whereby mitoses are aligned along the long axis of the duct. In this case, canonical and non-canonical Wnt signaling pathways seem to be involved in controlling the orientation of the plane cell division at different stages of duct elongation (Fischer et al. 2006; Karner et al. 2009).

\section{THE MAMMARY GLAND}

The mammary gland, like other glandular organs, contains a bilayered epithelial structure consisting of an outer layer of basal myoepithelial cells ensheathing an inner layer of luminal epithelial cells. Although the anlagen of the mammary gland are formed during embryonic stages, it is not until during postnatal mammary development that highly mitotic structures at the tips of growing ducts called "end buds" establish the mammary tree by invading the surrounding fatty stroma, which is composed of fibroblasts, adipocytes, nerves, blood vessels, and different immune cells ( for review, see Gjorevski and Nelson 2011). Although end bud bifurcation generates the primary architecture, lateral outgrowth of secondary and tertiary ducts is essential to achieve the full arborization of the fat pad. In contrast to all other branched structures we have thus far discussed, the branching pattern of the mammary gland is not stereotyped. However, and similar to other branched organs, mammary branching is also regulated by various signals from the epithelium or the stroma, including Fgfs, Bmps, Wnts, and epidermal growth factors (EGFs) (Brisken et al. 1998; Bocchinfuso et al. 2000; Hens and Wysolmerski 2005; Mallepell et al. 2006; Feng et al. 2007; Hens et al. 2007; Moraes et al. 2007). In addition, hormonal control plays a key role in triggering branching morphogenesis.

No signal has so far been identified that is specifically expressed by the stroma in regions that prefigure branch formation and outgrowth in the mammary gland. Thus, inhibitory signals might be critical to keep the branching program on hold, and transforming growth factor $\beta$ (TGF $\beta$ ) is a key negative regulator of the process. TGF $\beta$ has been proposed to exert this function by inducing the deposition of extracellular matrix and thereby decreasing branching (Pierce et al. 1993; Nelson et al. 2006). Interestingly, a recent study suggests that TGF $\beta$ induces the expression of roundabout 1 (Robo1) specifically in the basal layer, which then functions with Slit2 to limit branch formation by restricting basal cell proliferation via Wnt signaling (Macias et al. 2011). These studies propose that the specification of the number of basal cells is a critical component in the regulation of branch formation because of their role in releasing branching factors. Thus, Slit/Robo signaling represents a checkpoint to measure growth factor input by curbing basal cell proliferation.

The cell behavior underlying ductal elongation has recently been analyzed in organotypic 3D long-term cultures. 
Werb and colleagues showed that cells in elongating ducts reorganize into a multilayered epithelium, migrate collectively, and rearrange dynamically without forming cell protrusions (such as tracheal and endothelia tip cells), suggesting that branching morphogenesis involves active movement of both luminal and myoepithelial cell populations (Ewald et al. 2008).

\section{REGIONALIZATION OF BRANCHES DURING OUTGROWTH: GENERATION OF TIP VERSUS STALK}

One of the most interesting finding in the past few years regarding branching morphogenesis was the recognition that branches have an in-built polarity as they grow; they are subdivided into a tip and a stalk region (Fig. 2). The tip region can be thought as "the business end," where growth, guidance, and, in many cases, further branching are regulated; the stalk might also grow or extend as the tip grows, but in principle, the stalk temporarily consolidates the previously taken decision to grow a branch by firmly establishing it (although in some positions, the stalk cell phenotype can be redirected to a tip cell phenotype to reinitiate lateral branching). Early gene expression analyses and genetic analyses had already provided much evidence for such a tip versus stalk regionalization (e.g., Samakovlis et al. 1996a). More recently, it was shown that in both the trachea and in the vasculature, Notch/Delta signaling is intimately linked to this regionalization: high Notch signaling turning tip cell determination down, whereas high Bnl or Vegf signaling decreases Notch signaling by activating lateral inhibition through the up-regulation of Delta in cells closest to the source (see above). In the vasculature, the initiation of this Notch/Delta polarity might also be at the core of initial branch outgrowth and could represent a regulatory step involved in controlling where a branch will form. It is thus a balance between branch-promoting and branch-inhibiting factors that is tipped in one direction at branch points and in the other direction in the stalk, or in regions where branching is not initiated.

A second major finding was the realization that signaling pathways that guide neuronal extensions toward their targets (such as members of the Slit, Netrins, Ephrins, and Semaphorins) are intimately linked to branching morphogenesis in the trachea, the vasculature, and all of the other branched organs (for reviews, see Casanova 2007; Larrivee et al. 2009; Adams and Eichmann 2010). In the case of the vasculature, these molecules are clearly involved in the guidance of angiogenic sprouts or in the induction of sprouts; in the other branching systems, their role is less clear and needs to be defined more precisely. Whether these molecules act during branching morphogenesis to produce similar branching patterns, such as in the vasculature and the nervous system (guiding outgrowth), or because similar cellular targets are regulated (cytoskeletal elements, guidance signaling, etc.), remains to be seen. Because there are a limited number of signaling pathways at work in multicellular animals, it may not be surprising that most of them work in virtually all tissues.

\section{CONTROL OF CELL BEHAVIOR DURING THE BRANCHING PROCESS: A COMPARISON}

It is beyond doubt that the branching parameters-such as site and type of branching, branch angle, rate of elongation, change in tube diameter, and so on-are under the control of a cell fate determinant (e.g., Trachealess for the trachea) (Chung et al. 2011) and their interaction with signaling pathways (e.g., Fgf, Vegf, N) (see Affolter and Mann 2001), and that the two inputs collectively exert their function by regulating cell behavior. However, although our knowledge of molecular components has increased tremendously in the past decades, the exact contributions of distinct cellular activities to the formation of $3 \mathrm{D}$ branched structure are much less clear.

Cell migration is a driving force for branching in the trachea and the vasculature. Cell rearrangements are involved in the formation of all branched organs, and detailed descriptions of their important impact on branching have been obtained in the trachea. Similarly, cell shape changes are tightly linked to branching, but in many cases, it remains to be investigated whether these changes are the cause or the consequence of branching. In the tracheal system, cell migration causes cell intercalation as well as dramatic cell shape changes; although these cell shape changes contribute to the elongation of the branches and thus to the final architecture of the trachea, they might be obtained relatively passively as a consequence of other steps (for a more detailed description, see Caussinus et al. 2008). Directed cell division due to controlled spindle orientation has been shown to be very important for branch elongation in lung and kidney, although they might be controlled differently in the two systems (Tang et al. 2002; Fischer et al. 2006; Karner et al. 2009). Cell division is not involved in shaping the tracheal system from its invaginated epithelial bud; in all other cases we discussed, the structures grow tremendously during branching, and this growth in volume is brought about by cell division; although cell division has been argued not to be the driving force per se for lung and kidney branching, it is more likely to be an important determinant in mammary branching. Cell competition occurs in the trachea and the vasculature (controlled to a large extend via Notch/Delta lateral inhibition) and at sites of initial 
branch outgrowth in the mammary gland and the kidney. Clearly, cell competition (and the balance between branching or not; see above) needs to be further investigated and quantified because it represents a good candidate for a unifying mechanism contributing to branching morphogenesis, possibly by contributing to the tip/stalk subdivision of growing buds.

\section{BRANCH OUTGROWTH: REITERATION OF A BASIC PROCESS?}

It is, of course, hoped that once a branching event is understood at the molecular level in a given organ, all other branching events will follow a similar cellular logic within that organ, although regulated slightly differently at the molecular level to provide region-specific differences. This seems reasonable for those organs that are patterned relatively independent of other organs (lung, kidney, mammary gland). Indeed, a "branch generator" might be identified for each organ, and it will be extremely interesting to see whether this "branch generator" is similar or different in distinct organs. In the trachea and the vasculature, which extend throughout the entire body, the "branch generator" has been identified and relies on cell migration combined with cell competition (the Vegf/Fgf; Notch/Delta module); indeed, it is stunning how similarly the branching patterns are established in the invertebrate trachea and the vertebrate vasculature, and it will be interesting to see how many of the other branching parameters (e.g., rate of elongation, change in tube diameter, branch fusion) are controlled similarly in these two systems. Research into tracheal remodeling during larval and pupal stages in the fly might turn out to provide a system that is somewhat closer to the developing vasculature, because cell proliferation is also an essential part of the process (Guha et al. 2008; Sato et al. 2008; Weaver and Krasnow 2008).

In all other branched organs, it looks like RTK signaling is intimately linked to the branch generator. Feedback interactions (such as Fgf-Shh in the lung) (Fig. 4) might provide self-regulatory input into this branch generator (Affolter et al. 2009); this self-regulatory input might, in turn, be influenced locally via other components to give rise to a highly organized pattern. (Hox proteins and many other regionally expressed proteins influencing the formation of the body plan might feed into this process.)

\section{METHODS OF CHOICE TO UNDERSTAND BRANCHING MORPHOGENESIS}

Because branching implies changes in topology over time, it will be essential to use live imaging to investigate this process at cellular and subcellular resolution. Thus far, this has not been possible in many systems, explaining the lack of detailed knowledge regarding cell behavior in situ in wild-type organs as they undergo branching. Recent live imaging approaches have started to provide invaluable insight into the cellular basis of vascular development using the zebrafish embryo and tracheal development in the fly embryo (e.g., Caussinus et al. 2008; Herwig et al. 2011). Similar approaches will have to be developed to image the development of the lung, the kidney, and the mammary gland in vivo.

Cell tracking will become more important and has already been successfully used in the kidney. Cell tracking using mosaic analyses based on methods such as Brainbow (Livet et al. 2007) might be crucial to follow large groups of cells during morphogenesis. In the trachea and the vasculature, such analyses were not as important because individual cells could easily be followed using live imaging; in other organs, which are made up of many more cells, this will not be easily possible, and generating inducible mosaics will help in providing better insight at the cellular level.

Changes in shape (at the cellular or tissue level) or, more generally, morphogenesis processes at large, are often brought about by forces. In the future, it will be very important to map forces during branching morphogenesis and find out whether they are the cause or the consequence of branching. Cells actively engaged in force generation and the cytoskeletal elements and motor proteins responsible for this will have to be identified. A key challenge will then be to connect these findings to the higher-order shape generation at the tissue level. Quite obviously, competitive interactions in groups of cells in combination with the generation of forces and responses to these forces might result in non-intuitive global tissue behavior. Mathematical modeling at all levels, from gene regulation to global tissue behaviors, is increasingly helping us to understand complex processes during morphogenesis and is putting forward hypotheses that can be tested experimentally.

A nice example of computational modeling on global tissue behaviors comes from the work of Sharpe and colleagues, who generated an extensive data set of 3D shape changes and proliferation rates for the limb bud and with this data tested the long-standing growth-based morphogenesis hypothesis of limb bud elongation (Fig. 5) (Boehm et al. 2010). This hypothesis suggests that directional elongation is the result of faster proliferation rates in proximal cells. 3D simulation of their quantitative data showed that graded non-oriented cell proliferation cannot explain limb bud elongation, and that other directional cell activities might be responsible for the outgrowth. This new hypothesis led them to identify directional cell behaviors in the 
1) 3D growth pattern

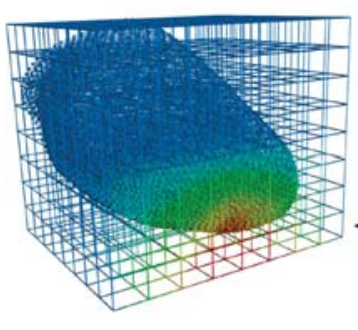

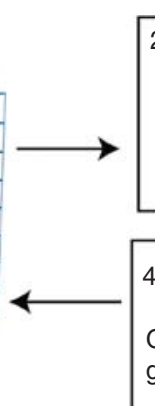

2) FEM simulation Predicts shapechange

4) Parameter optimization

Generates new growth pattern
3) Shape comparison

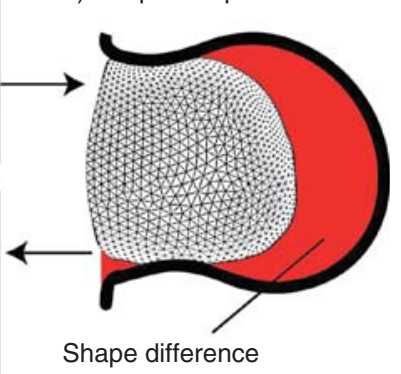

Figure 5. Limb development modeling through parameter exploration. Boehm et al. (2010) mapped theoretical proliferation values onto the observed initial limb shape (1). Using these values, the simulation produces a final limb shape that is compared with the observed final limb shape $(2,3)$; a parameter optimization step (4) generates a new initial proliferation pattern (1) that goes through the optimization process until a stable proliferation pattern is achieved. The simulations either fail or succeed to recapitulate the observed limb growth and the theoretical values, giving rise to a given shape that can be compared with the observed experimental data. (Reproduced from PLoS Biol., Boehm et al. 2010.)

bud mesenchyme that might be responsible for limb bud elongation (dynamically extending and retracting filopodia, distal positioning of the Golgi and bias in the orientation cell division) (Boehm et al. 2010). Cell tracking and computational modeling have also been successfully used to study several other developmental processes (see Benazeraf et al. 2010; Gros et al. 2010).

The computational simulation of gene regulatory network behavior during organ formation will be very useful to understand the molecular interactions underlying branching morphogenesis. Menshykau et al. (2012) took precisely this approach and modeled the gene regulatory network described for lung growth and patterning (Fig. 4). Considering the most relevant players (Fgf10, Shh, and Ptc), their interactions and distribution within the lung bud, as well as experimental parameter values, they developed a two-dimensional (2D) Turing-based model that can reproduce the available lung branching data for both wildtype and a number of mutant mice. By exploring different ranges of parameters, two steady-state patterns of Fgf10 emerged in the context of a lung-shaped domain: one centered at the tip of the lung bud and the second one concentrated on the sides of the tip. These two patterns are likely to correspond to two different types of branching; Fgf10 concentrated at the very tip giving rise to an elongating bud that branches laterally, and Fgf10 on the sides giving rise to bifurcations. Menshykau et al. (2012) showed that almost any parameter change can generate the two Fgf10 steady states that could give rise to different branching modes. An interesting parameter, however, growth rate, also altered the branching patterns in their simulations: fast growth triggers lateral branching, whereas slow growth favors branch bifurcations. The investigators speculate that
Fgf10 concentration itself could affect the growth rate such that changes in its concentration during growth would determine the sequence of branching events (Menshykau et al. 2012).

Many more gene regulatory networks involved in morphogenetic processes have been described, including positive and negative feedback loops. These interactions can be modeled using different approaches. Given the great amount of experimental data relating to these networks, it is feasible to readily test the predictions of such models, eventually moving toward a more quantitative understanding of morphogenesis. At the end of the day, cell and tissue behaviors will have to be linked to the underlying gene regulatory network; only then will we have a plausible explanation for a branching process that satisfies both computer modelers and biologists equally. If we take into account the progress that has been made in the past 10 years, we are reinforced in our belief that this might happen in the not-too-distant future. We fully agree with the statement of Little and Wieschaus: “... studies of morphogenesis should aim to unify observations on a variety of time and length scale to thus understand how temporally and spatially restricted molecular activities give rise to the marvellous patterns of morphogenesis observed in diverse organisms" (Little and Wieschaus 2011).

\section{ACKNOWLEDGMENTS}

We thank Ross Metzger, Magdalena Baer, and Dagmar Iber for critical reading of the manuscript. Funding is from SystemsX.ch within the framework of the WingX RTD, the Swiss National Science Foundation, the Kantons of Basel-Stadt and Basel-Land. 


\section{REFERENCES}

Adams RH, Eichmann A. 2010. Axon guidance molecules in vascular patterning. Cold Spring Harb Perspect Biol 2: a001875.

Affolter M, Caussinus E. 2008. Tracheal branching morphogenesis in Drosophila: New insights into cell behaviour and organ architecture. Development 135: 2055-2064.

Affolter M, Mann R. 2001. Development. Legs, eyes, or wings—selectors and signals make the difference. Science 292: 1080-1081.

Affolter M, Zeller R, Caussinus E. 2009. Tissue remodelling through branching morphogenesis. Nat Rev Mol Cell Biol 10: 831-842.

Andrew DJ, Ewald AJ. 2010. Morphogenesis of epithelial tubes: Insights into tube formation, elongation, and elaboration. Dev Biol 341: $34-55$.

Bellusci S, Henderson R, Winnier G, Oikawa T, Hogan BL. 1996. Evidence from normal expression and targeted misexpression that bone morphogenetic protein (Bmp-4) plays a role in mouse embryonic lung morphogenesis. Development 122: 1693-1702.

Bellusci S, Furuta Y, Rush MG, Henderson R, Winnier G, Hogan BL. 1997a. Involvement of Sonic hedgehog (Shh) in mouse embryonic lung growth and morphogenesis. Development 124: 53-63.

Bellusci S, Grindley J, Emoto H, Itoh N, Hogan BL. 1997b. Fibroblast growth factor 10 (FGF10) and branching morphogenesis in the embryonic mouse lung. Development 124: 4867-4878.

Benazeraf B, Francois P, Baker RE, Denans N, Little CD, Pourquie O. 2010. A random cell motility gradient downstream of FGF controls elongation of an amniote embryo. Nature 466: 248-252.

Bocchinfuso WP, Lindzey JK, Hewitt SC, Clark JA, Myers PH, Cooper R, Korach KS. 2000. Induction of mammary gland development in estrogen receptor- $\alpha$ knockout mice. Endocrinology 141: 2982-2994.

Boehm B, Westerberg H, Lesnicar-Pucko G, Raja S, Rautschka M, Cotterell J, Swoger J, Sharpe J. 2010. The role of spatially controlled cell proliferation in limb bud morphogenesis. PLoS Biol 8: e1000420.

Brisken C, Park S, Vass T, Lydon JP, O’Malley BW, Weinberg RA. 1998. A paracrine role for the epithelial progesterone receptor in mammary gland development. Proc Natl Acad Sci 95: 5076-5081.

Burri PH, Hlushchuk R, Djonov V. 2004. Intussusceptive angiogenesis: Its emergence, its characteristics, and its significance. Dev Dyn 231: 474-488.

Cabernard C, Affolter M. 2005. Distinct roles for two receptor tyrosine kinases in epithelial branching morphogenesis in Drosophila. Dev Cell 9: $831-842$.

Casanova J. 2007. The emergence of shape: Notions from the study of the Drosophila tracheal system. EMBO Rep 8: 335-339.

Caussinus E, Colombelli J, Affolter M. 2008. Tip-cell migration controls stalk-cell intercalation during Drosophila tracheal tube elongation. Curr Biol 18: 1727-1734.

Chi X, Michos O, Shakya R, Riccio P, Enomoto H, Licht JD, Asai N, Takahashi M, Ohgami N, Kato M, et al. 2009. Ret-dependent cell rearrangements in the Wolffian duct epithelium initiate ureteric bud morphogenesis. Dev Cell 17: 199-209.

Chung S, Chavez C, Andrew DJ. 2011. Trachealess (Trh) regulates all tracheal genes during Drosophila embryogenesis. Dev Biol 360: 160-172.

Costantini F. 2006. Renal branching morphogenesis: Concepts, questions, and recent advances. Differentiation 74: 402-421.

Costantini F, Kopan R. 2010. Patterning a complex organ: Branching morphogenesis and nephron segmentation in kidney development. Dev Cell 18: 698-712.

Costantini F, Shakya R. 2006. GDNF/Ret signaling and the development of the kidney. BioEssays 28: 117-127.

Coultas L, Chawengsaksophak K, Rossant J. 2005. Endothelial cells and VEGF in vascular development. Nature 438: 937-945.

De Moerlooze L, Spencer-Dene B, Revest JM, Hajihosseini M, Rosewell I, Dickson C. 2000. An important role for the IIIb isoform of fibroblast growth factor receptor 2 (FGFR2) in mesenchymal-epithelial signalling during mouse organogenesis. Development 127: 483-492.

Eblaghie MC, Reedy M, Oliver T, Mishina Y, Hogan BL. 2006. Evidence that autocrine signaling through Bmprla regulates the proliferation, survival and morphogenetic behavior of distal lung epithelial cells. Dev Biol 291: 67-82.

Ewald AJ, Brenot A, Duong M, Chan BS, Werb Z. 2008. Collective epithelial migration and cell rearrangements drive mammary branching morphogenesis. Dev Cell 14: 570-581.

Feng Y, Manka D, Wagner KU, Khan SA. 2007. Estrogen receptor- $\alpha$ expression in the mammary epithelium is required for ductal and alveolar morphogenesis in mice. Proc Natl Acad Sci 104: 14718-14723.

Ferrara N, Gerber HP, LeCouter J. 2003. The biology of VEGF and its receptors. Nat Med 9: 669-676.

Fischer E, Legue E, Doyen A, Nato F, Nicolas JF, Torres V, Yaniv M, Pontoglio M. 2006. Defective planar cell polarity in polycystic kidney disease. Nat Genet 38: 21-23.

Franch-Marro X, Casanova J. 2000. The alternative migratory pathways of the Drosophila tracheal cells are associated with distinct subsets of mesodermal cells. Dev Biol 227: 80-90.

Gerhardt H, Golding M, Fruttiger M, Ruhrberg C, Lundkvist A, Abramsson A, Jeltsch M, Mitchell C, Alitalo K, Shima D, et al. 2003. VEGF guides angiogenic sprouting utilizing endothelial tip cell filopodia. J Cell Biol 161: 1163-1177.

Gervais L, Casanova J. 2010. In vivo coupling of cell elongation and lumen formation in a single cell. Curr Biol 20: 359-366.

Geudens I, Gerhardt H. 2011. Coordinating cell behaviour during blood vessel formation. Development 138: 4569-4583.

Ghabrial AS, Krasnow MA. 2006. Social interactions among epithelial cells during tracheal branching morphogenesis. Nature 441: 746-749.

Ghabrial A, Luschnig S, Metzstein MM, Krasnow MA. 2003. Branching morphogenesis of the Drosophila tracheal system. Annu Rev Cell Dev Biol 19: 623-647.

Ghabrial AS, Levi BP, Krasnow MA. 2011. A systematic screen for tube morphogenesis and branching genes in the Drosophila tracheal system. PLoS Genet 7: e1002087.

Gibson DA, Ma L. 2011. Developmental regulation of axon branching in the vertebrate nervous system. Development 138: 183-195.

Gjorevski N, Nelson CM. 2011. Integrated morphodynamic signalling of the mammary gland. Nat Rev Mol Cell Biol 12: 581-593.

Gros J, Hu JK, Vinegoni C, Feruglio PF, Weissleder R, Tabin CJ. 2010. WNT5A/JNK and FGF/MAPK pathways regulate the cellular events shaping the vertebrate limb bud. Curr Biol 20: 1993-2002.

Grueber WB, Sagasti A. 2010. Self-avoidance and tiling: Mechanisms of dendrite and axon spacing. Cold Spring Harb Perspect Biol 2: a001750.

Guha A, Lin L, Kornberg TB. 2008. Organ renewal and cell divisions by differentiated cells in Drosophila. Proc Natl Acad Sci 105: 1083210836.

Hellstrom M, Phng LK, Hofmann JJ, Wallgard E, Coultas L, Lindblom P, Alva J, Nilsson AK, Karlsson L, Gaiano N, et al. 2007. Dll4 signalling through Notch1 regulates formation of tip cells during angiogenesis. Nature 445: 776-780.

Hens JR, Wysolmerski JJ. 2005. Key stages of mammary gland development: Molecular mechanisms involved in the formation of the embryonic mammary gland. Breast Cancer Res 7: 220-224.

Hens JR, Dann P, Zhang JP, Harris S, Robinson GW, Wysolmerski J. 2007. BMP4 and PTHrP interact to stimulate ductal outgrowth during embryonic mammary development and to inhibit hair follicle induction. Development 134: 1221-1230.

Herwig L, Blum Y, Krudewig A, Ellertsdottir E, Lenard A, Belting HG, Affolter M. 2011. Distinct cellular mechanisms of blood vessel fusion in the zebrafish embryo. Curr Biol 21: 1942-1948.

Jan YN, Jan LY. 2010. Branching out: Mechanisms of dendritic arborization. Nat Rev Neurosci 11: 316-328.

Karner CM, Chirumamilla R, Aoki S, Igarashi P, Wallingford JB, Carroll TJ. 2009. Wnt9b signaling regulates planar cell polarity and kidney tubule morphogenesis. Nat Genet 41: 793-799.

Kim J, Oh WJ, Gaiano N, Yoshida Y, Gu C. 2011. Semaphorin 3E-PlexinD1 signaling regulates VEGF function in developmental angiogenesis via a feedback mechanism. Genes Dev 25: 1399-1411. 
Klambt C, Glazer L, Shilo BZ. 1992. breathless, a Drosophila FGF receptor homolog, is essential for migration of tracheal and specific midline glial cells. Genes Dev 6: 1668-1678.

Larrivee B, Freitas C, Suchting S, Brunet I, Eichmann A. 2009. Guidance of vascular development: Lessons from the nervous system. Circ Res 104: $428-441$.

Lazarus A, Del-Moral PM, Ilovich O, Mishani E, Warburton D, Keshet E. 2011. A perfusion-independent role of blood vessels in determining branching stereotypy of lung airways. Development 138: 2359-2368.

Lebeche D, Malpel S, Cardoso WV. 1999. Fibroblast growth factor interactions in the developing lung. Mech Dev 86: 125-136.

Leslie JD, Ariza-McNaughton L, Bermange AL, McAdow R, Johnson SL, Lewis J. 2007. Endothelial signalling by the Notch ligand Delta-like 4 restricts angiogenesis. Development 134: 839-844.

Leung DW, Cachianes G, Kuang WJ, Goeddel DV, Ferrara N. 1989. Vascular endothelial growth factor is a secreted angiogenic mitogen. Science 246: 1306-1309.

Lim AH, Suli A, Yaniv K, Weinstein B, Li DY, Chien CB. 2011. Motoneurons are essential for vascular pathfinding. Development 138: $3847-$ 3857.

Little SC, Wieschaus EF. 2011. Shifting patterns: Merging molecules, morphogens, motility, and methodology. Dev Cell 21: 2-4.

Liu ZJ, Shirakawa T, Li Y, Soma A, Oka M, Dotto GP, Fairman RM, Velazquez OC, Herlyn M. 2003. Regulation of Notch1 and Dll4 by vascular endothelial growth factor in arterial endothelial cells: Implications for modulating arteriogenesis and angiogenesis. Mol Cell Biol 23: $14-25$.

Liu Y, Stein E, Oliver T, Li Y, Brunken WJ, Koch M, Tessier-Lavigne M, Hogan BL. 2004. Novel role for Netrins in regulating epithelial behavior during lung branching morphogenesis. Curr Biol 14: 897-905.

Livet J, Weissman TA, Kang H, Draft RW, Lu J, Bennis RA, Sanes JR, Lichtman JW. 2007. Transgenic strategies for combinatorial expression of fluorescent proteins in the nervous system. Nature 450: $56-62$

Lobov IB, Renard RA, Papadopoulos N, Gale NW, Thurston G, Yancopoulos GD, Wiegand SJ. 2007. Delta-like ligand 4 (Dll4) is induced by VEGF as a negative regulator of angiogenic sprouting. Proc Natl Acad Sci 104: 3219-3224.

Lohela M, Bry M, Tammela T, Alitalo K. 2009. VEGFs and receptors involved in angiogenesis versus lymphangiogenesis. Curr Opin Cell Biol 21: 154-165.

Lu P, Werb Z. 2008. Patterning mechanisms of branched organs. Science 322: $1506-1509$.

Lu X, Le Noble F, Yuan L, Jiang Q, De Lafarge B, Sugiyama D, Breant C, Claes F, De Smet F, Thomas JL, et al. 2004. The netrin receptor UNC5B mediates guidance events controlling morphogenesis of the vascular system. Nature 432: 179-186.

Luschnig S, Batz T, Armbruster K, Krasnow MA. 2006. serpentine and vermiform encode matrix proteins with chitin binding and deacetylation domains that limit tracheal tube length in Drosophila. Curr Biol 16: $186-194$

Macias H, Moran A, Samara Y, Moreno M, Compton JE, Harburg G, Strickland P, Hinck L. 2011. SLIT/ROBO1 signaling suppresses mammary branching morphogenesis by limiting basal cell number. Dev Cell 20: 827-840.

Mailleux AA, Tefft D, Ndiaye D, Itoh N, Thiery JP, Warburton D, Bellusci S. 2001. Evidence that SPROUTY2 functions as an inhibitor of mouse embryonic lung growth and morphogenesis. Mech Dev 102: $81-94$.

Makanya AN, Hlushchuk R, Djonov VG. 2009. Intussusceptive angiogenesis and its role in vascular morphogenesis, patterning, and remodeling. Angiogenesis 12: 113-123.

Mallepell S, Krust A, Chambon P, Brisken C. 2006. Paracrine signaling through the epithelial estrogen receptor alpha is required for proliferation and morphogenesis in the mammary gland. Proc Natl Acad Sci 103: $2196-2201$.
Maruyama R, Andrew DJ. 2012. Drosophila as a model for epithelial tube formation. Dev Dyn 241: 119-135.

Menshykau D, Kraemer C, Iber D. 2012. Branch mode selection during early lung development. PLoS Comput Biol 8: e1002377.

Metzger RJ, Klein OD, Martin GR, Krasnow MA. 2008. The branching programme of mouse lung development. Nature 453: 745-750.

Michael L, Davies JA. 2004. Pattern and regulation of cell proliferation during murine ureteric bud development. J Anat 204: 241-255.

Michos O. 2009. Kidney development: From ureteric bud formation to branching morphogenesis. Curr Opin Genet Dev 19: 484-490.

Michos O, Cebrian C, Hyink D, Grieshammer U, Williams L, D’Agati V, Licht JD, Martin GR, Costantini F. 2010. Kidney development in the absence of Gdnf and Spry1 requires Fgf10. PLoS Genet 6: e1000809.

Moraes RC, Zhang X, Harrington N, Fung JY, Wu MF, Hilsenbeck SG, Allred DC, Lewis MT. 2007. Constitutive activation of smoothened (SMO) in mammary glands of transgenic mice leads to increased proliferation, altered differentiation and ductal dysplasia. Development 134: 1231-1242.

Morrisey EE, Hogan BL. 2010. Preparing for the first breath: Genetic and cellular mechanisms in lung development. Dev Cell 18: 8-23.

Mucenski ML, Wert SE, Nation JM, Loudy DE, Huelsken J, Birchmeier W, Morrisey EE, Whitsett JA. 2003. $\beta$-Catenin is required for specification of proximal/distal cell fate during lung morphogenesis. J Biol Chem 278: $40231-40238$.

Mukouyama YS, Gerber HP, Ferrara N, Gu C, Anderson DJ. 2005. Peripheral nerve-derived VEGF promotes arterial differentiation via neuropilin 1-mediated positive feedback. Development 132: 941-952.

Nelson CM, Vanduijn MM, Inman JL, Fletcher DA, Bissell MJ. 2006. Tissue geometry determines sites of mammary branching morphogenesis in organotypic cultures. Science 314: 298-300.

Pierce DF Jr, Johnson MD, Matsui Y, Robinson SD, Gold LI, Purchio AF, Daniel CW, Hogan BL, Moses HL. 1993. Inhibition of mammary duct development but not alveolar outgrowth during pregnancy in transgenic mice expressing active TGF- $\beta 1$. Genes Dev 7: 2308-2317.

Risau W. 1997. Mechanisms of angiogenesis. Nature 386: 671-674.

Ruhrberg C. 2003. Growing and shaping the vascular tree: Multiple roles for VEGF. BioEssays 25: 1052-1060.

Samakovlis C, Hacohen N, Manning G, Sutherland DC, Guillemin K, Krasnow MA. 1996a. Development of the Drosophila tracheal system occurs by a series of morphologically distinct but genetically coupled branching events. Development 122: 1395-1407.

Samakovlis C, Manning G, Steneberg P, Hacohen N, Cantera R, Krasnow MA. 1996b. Genetic control of epithelial tube fusion during Drosophila tracheal development. Development 122: 3531-3536.

Sariola H, Saarma M. 2003. Novel functions and signalling pathways for GDNF. J Cell Sci 116: 3855-3862.

Sato M, Kitada Y, Tabata T. 2008. Larval cells become imaginal cells under the control of homothorax prior to metamorphosis in the Drosophila tracheal system. Dev Biol 318: 247-257.

Schedin P, O’Brien J, Rudolph M, Stein T, Borges V. 2007. Microenvironment of the involuting mammary gland mediates mammary cancer progression. J Mammary Gland Biol Neoplasia 12: 71-82.

Schottenfeld J, Song Y, Ghabrial AS. 2010. Tube continued: Morphogenesis of the Drosophila tracheal system. Curr Opin Cell Biol 22: 633-639.

Sekine K, Ohuchi H, Fujiwara M, Yamasaki M, Yoshizawa T, Sato T, Yagishita N, Matsui D, Koga Y, Itoh N, et al. 1999. Fgf10 is essential for limb and lung formation. Nat Genet 21: 138-141.

Shakya R, Watanabe T, Costantini F. 2005. The role of GDNF/Ret signaling in ureteric bud cell fate and branching morphogenesis. Dev Cell 8: 65-74.

Shu W, Guttentag S, Wang Z, Andl T, Ballard P, Lu MM, Piccolo S, Birchmeier W, Whitsett JA, Millar SE, et al. 2005. Wnt $/ \beta$-catenin signaling acts upstream of N-myc, BMP4, and FGF signaling to regulate proximal-distal patterning in the lung. Dev Biol 283: 226-239. 
Shutter JR, Scully S, Fan W, Richards WG, Kitajewski J, Deblandre GA, Kintner CR, Stark KL. 2000. Dll4, a novel Notch ligand expressed in arterial endothelium. Genes Dev 14: 1313-1318.

Siekmann AF, Lawson ND. 2007. Notch signalling limits angiogenic cell behaviour in developing zebrafish arteries. Nature 445: 781-784.

Suchting S, Freitas C, le Noble F, Benedito R, Breant C, Duarte A, Eichmann A. 2007. The Notch ligand Delta-like 4 negatively regulates endothelial tip cell formation and vessel branching. Proc Natl Acad Sci 104: $3225-3230$

Sutherland D, Samakovlis C, Krasnow MA. 1996. branchless encodes a Drosophila FGF homolog that controls tracheal cell migration and the pattern of branching. Cell 87: 1091-1101.

Swift MR, Weinstein BM. 2009. Arterial-venous specification during development. Circ Res 104: 576-588.

Tang MJ, Cai Y, Tsai SJ, Wang YK, Dressler GR. 2002. Ureteric bud outgrowth in response to RET activation is mediated by phosphatidylinositol 3-kinase. Dev Biol 243: 128-136.

Tang N, Marshall WF, McMahon M, Metzger RJ, Martin GR. 2011. Control of mitotic spindle angle by the RAS-regulated ERK1/2 pathway determines lung tube shape. Science 333: 342-345.

Tefft JD, Lee M, Smith S, Leinwand M, Zhao J, Bringas P Jr, Crowe DL, Warburton D. 1999. Conserved function of mSpry-2, a murine homolog of Drosophila sprouty, which negatively modulates respiratory organogenesis. Curr Biol 9: 219-222.

Tsarouhas V, Senti KA, Jayaram SA, Tiklova K, Hemphala J, Adler J, Samakovlis C. 2007. Sequential pulses of apical epithelial secretion and endocytosis drive airway maturation in Drosophila. Dev Cell 13: 214-225.

Uv A, Cantera R, Samakovlis C. 2003. Drosophila tracheal morphogenesis: Intricate cellular solutions to basic plumbing problems. Trends Cell Biol 13: 301-309.

Wacker A, Gerhardt H. 2011. Endothelial development taking shape. Curr Opin Cell Biol 23: 676-685.

Wang S, Jayaram SA, Hemphala J, Senti KA, Tsarouhas V, Jin H, Samakovlis C. 2006. Septate-junction-dependent luminal deposition of chitin deacetylases restricts tube elongation in the Drosophila trachea. Curr Biol 16: 180-185.

Watanabe T, Costantini F. 2004. Real-time analysis of ureteric bud branching morphogenesis in vitro. Dev Biol 271: 98-108.

Weaver M, Krasnow MA. 2008. Dual origin of tissue-specific progenitor cells in Drosophila tracheal remodeling. Science 321: 14961499.

Williams CK, Li JL, Murga M, Harris AL, Tosato G. 2006. Up-regulation of the Notch ligand Delta-like 4 inhibits VEGF-induced endothelial cell function. Blood 107: 931-939.

Zeller R, Lopez-Rios J, Zuniga A. 2009. Vertebrate limb bud development: Moving towards integrative analysis of organogenesis. Nat Rev Genet 10: 845-858.

Zygmunt T, Gay CM, Blondelle J, Singh MK, Flaherty KM, Means PC, Herwig L, Krudewig A, Belting HG, Affolter M, et al. 2011. Semaphorin-PlexinD1 signaling limits angiogenic potential via the VEGF decoy receptor sFlt1. Dev Cell 21: 301-314. 


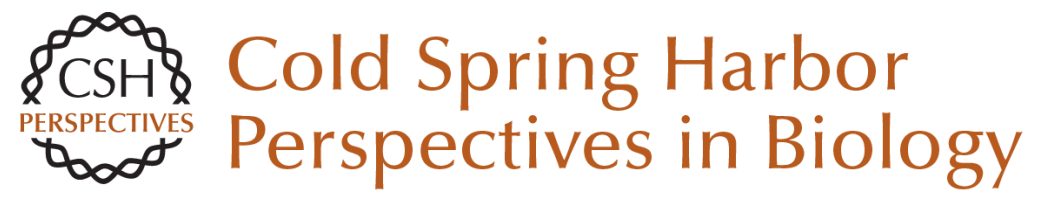

\section{Branching Morphogenesis: From Cells to Organs and Back}

Amanda Ochoa-Espinosa and Markus Affolter

Cold Spring Harb Perspect Biol 2012; doi: 10.1101/cshperspect.a008243 originally published online July 12,2012

\section{Subject Collection Mammalian Development}

The Dynamics of Morphogenesis in the Early Mouse Embryo

Jaime A. Rivera-Pérez and Anna-Katerina Hadjantonakis

microRNAs as Developmental Regulators Kathryn N. Ivey and Deepak Srivastava

Development of the Endochondral Skeleton Fanxin Long and David M. Ornitz

\section{Adipogenesis}

Kelesha Sarjeant and Jacqueline M. Stephens

Molecular Mechanisms of Inner Ear Development Doris K. Wu and Matthew W. Kelley

Polarity in Mammalian Epithelial Morphogenesis Julie Roignot, Xiao Peng and Keith Mostov

Eye Development and Retinogenesis Whitney Heavner and Larysa Pevny

Primordial Germ Cells in Mice Mitinori Saitou and Masashi Yamaji
Cell Division Modes and Cleavage Planes of Neural Progenitors during Mammalian Cortical Development

Fumio Matsuzaki and Atsunori Shitamukai

Blood and Lymphatic Vessel Formation Victoria L. Bautch and Kathleen M. Caron

Transcriptional Networks in Liver and Intestinal Development

Karyn L. Sheaffer and Klaus H. Kaestner

Pluripotency in the Embryo and in Culture Jennifer Nichols and Austin Smith

Signaling and Transcriptional Networks in Heart Development and Regeneration Benoit G. Bruneau

Signals and Switches in Mammalian Neural Crest Cell Differentiation

Shachi Bhatt, Raul Diaz and Paul A. Trainor

Hematopoiesis Michael A. Rieger and Timm Schroeder

Intercellular Interactions, Position, and Polarity in Establishing Blastocyst Cell Lineages and

Embryonic Axes Robert O. Stephenson, Janet Rossant and Patrick P.L. Tam

For additional articles in this collection, see http://cshperspectives.cshlp.org/cgi/collection/

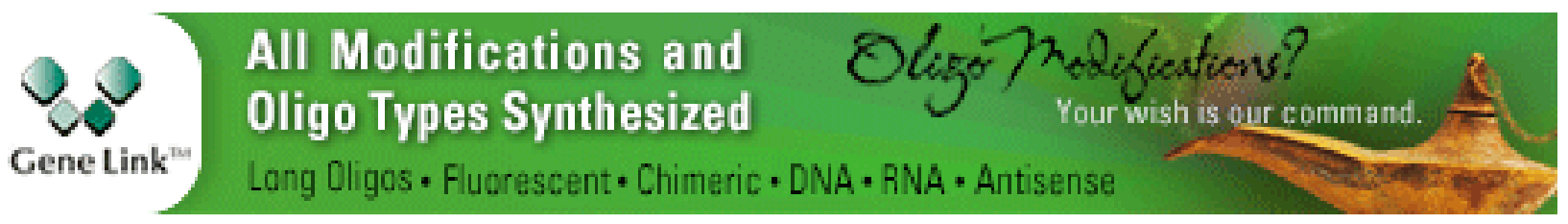

Copyright @ 2012 Cold Spring Harbor Laboratory Press; all rights reserved 